\title{
Infra-red studies of dwarf galaxies in the Fornax cluster
}

\author{
Rachel Smith, Steve Phillipps \\ and Roberto De Propris
}

H.H. Wills Physics Laboratory, University of Bristol, Tyndall Avenue, Bristol, BS8 1TL email: rachel.smith@bristol.ac.uk

\section{Introduction}

Dwarf galaxies are the most numerous type of galaxy in the local universe and are extremely important in the understanding of galaxy formation and evolution. A better understanding of the physics of dwarf galaxies is central to several current issues in observational cosmology. For example, simulations of galaxy evolution based on the ColdDark-Matter (CDM) model predict far more dwarfs than are observed locally. In the last 20 years there has been a huge amount of interest in the study of dwarf galaxies, partly due to these unanswered puzzles and also because of the improvements in the technology available.

One area into which we are researching involves analysing the follow-up infra-red (IR) data from the Fornax Cluster Spectroscopic Survey (FCSS). The FCSS is an all-object survey of a region around the Fornax Cluster of galaxies (Drinkwater et al. 2000 and Phillipps et al. 2001). The aim of the FCSS was to obtain spectra for a complete sample of all objects within the magnitude range $16.5 \leqslant b_{j} \leqslant 19.7$, irrespective of their image morphology. We have used this to obtain a well defined sample of cluster dwarfs.

\section{Infra-red Observations}

IR observations are a very useful tool in studying the astrophysical nature of dwarf galaxies. Near IR luminosities are generally regarded as a better surrogate for mass, than those at shorter wavelengths, which are more influenced by recent star formation. In particular this allows us to consider global properties such as the overall baryonic (or at least stellar) mass and the mass surface density.

We are interested in answering several different questions about Fornax Cluster dwarfs: Are they all old? Are nucleated dwarfs older than non-nucleated dwarfs? Are the ultracompact dwarfs (UCDs) a different age to the other dwarfs, and what would this tell us about their evolution? We are also interested in the metallicity of these galaxies. For a few of the dwarfs we have spectroscopic data, however most will be investigated using broad-band colours.

The IR data we are using consists of observations of a sample of Fornax cluster dwarf galaxies in the $\mathrm{K}^{\prime}$ band, taken in November 2000 using the Cryogenic Array Spectrometer/Imager (CASPIR) on the $2.3 \mathrm{~m}$ telescope at Siding Spring Observatory and $\mathrm{K}_{s}$ band observations of a separate sample taken in December 2002 using the 3.6m ESO-New Technology Telescope (NTT) equipped with the Son of ISAAC (SOFI) infrared spectrograph and imaging camera. 


\section{Stellar Population Models}

There are complications in using broad-band colours to model galaxy properties. As well as stellar evolution, chemical evolution in stars also affects the colour of the galaxy, which causes an age/metallicity degeneracy problem. Stars with high metallicities appear redder than those which are under abundant in heavy elements. It is therefore difficult to separate populations which have a combination of age and metallicity effects; the optical colours of composite stellar populations are virtually identical if the percentage change in age or metallicity $(\mathrm{Z})$ follows $\Delta$ age $/ \Delta \mathrm{Z} \sim 3 / 2$ (Worthey 1994). IR data is invaluable in disentangling age and metallicity effects in the star formation histories. By combining NIR data with optical colours we can constrain stellar populations by using stellar population synthesis (SPS) models.

Several different SPS models are available and their individual strengths and weaknesses have been discussed in the literature (e.g. Worthey 1994 and Bell \& de Jong 2000). We are working with collaborators at the Astrophysics Research Institute, Liverpool John Moores University, who are developing their own predictions of the optical - NIR colours of stellar populations as a function of age and metallicity, using models developed by M. Salaris and S. Cassisi. They have identified the (B-K) - (J-K) colour-colour plane as particularly sensitive for breaking age-metallicity degeneracy. It is found that the integrated colour ( J-K) appears to be a good tracer of the initial metallicity of a simple stellar population (SSP) and is weakly affected by age, whereas the integrated $(\mathrm{B}-\mathrm{K})$ is a good age indicator, mildly affected by the SSP metallicity (James et al. in prep.).

\section{Current Progress and Future Work}

We have high signal/noise reduced images for 9 Fornax Cluster dwarfs. Surface brightness profiles and K-band magnitudes will be measured from these images. In most cases these can be calibrated directly from the final image, as the majority of the galaxies have 2MASS stars within the object frame.

Using this IR data, alongside existing optical data, we will investigate morphological and structural properties, star formation rates and histories, and stellar populations and their metallicities in dwarf galaxies. A comparison of the broad-band colours and spectal methods of obtaining metallicities can be made, as both types of data are available for a few of our objects.

\section{References}

Bell, E.F. \& de Jong, R.S. 2000, MNRAS 312, 497

Drinkwater, M.J., Phillipps, S., Jones, J.B., Gregg, M.D., Deady, J.H., Davies, J.I., Parker, Q.A., Sadler, E.M. \& Smith, R.M. 2000, A\&A A 355, 900

James, P.A., Salaris, M., Davies, J.I., Phillipps, S. \& Cassisi, S. In prep.

Phillipps, S., Drinkwater, M.J., Gregg, M.D. \& Jones, J.B. 2001 ApJ 560, 201

Worthey, G. 1994, ApJS 95, 107 